\title{
Acoustic full waveform inversion for sub-seafloor e xploration using vertical cable seismic
}

\author{
Ehsan JAMALI HONDORI ${ }^{1}$, Hitoshi MIKADA ${ }^{2}$, Eiichi ASAKAWA ${ }^{1}$, \\ and Shigeharu MIZOHATA ${ }^{1}$ \\ ${ }^{1}$ Innovative Technology Dept., JGI Inc. \\ ${ }^{2}$ Dept. of Civil and Earth Res. Eng., Kyoto University
}

\begin{abstract}
Full waveform inversion (FWI) of seismic reflection data has been widely used with different 2D/3D data acquisition geometries. The method develops a reliable subsurface model by minimizing misfit between observed and simulated waveforms. Vertical Cable Seismic (VCS) is a recent seismic reflection method which uses vertical array of hydrophones in order to record acoustic energy generated by seismic sources. VCS can acquire high resolution data by deploying hydrophone arrays very near to the seafloor which results in a higher signal to noise ratio. Because of the especial geometry of VCS data it is challenging to develop a velocity model based on the conventional processing techniques. We used a simulation experiment to evaluate the FWI results on seismic reflection data acquired using VCS geometry. Although dominant events in the data were reflections rather than diving waves, which are important for FWI, we could obtain a promising velocity model.
\end{abstract}

\section{INTRODUCTION}

Subsurface exploration for natural resources is mainly conducted by surface seismic surveys which use receiver arrays on the surface to record the energy of acoustic waves propagated from seismic sources and produce an image of the earth after data processing for the acquired waves scattered in the subsurface. Sources and receivers could be placed on the ground, land acquisition, or towed in the sea by a vessel, marine acquisition, in order to cover the desired area of the imaging. This requires appropriate data acquisition geometry and optimized data processing workflows for reliable subsurface images. However, the surface seismic exploration fails to deliver a high resolution image in the marine environments with certain obstacles like platforms or buoys, in the deep sea conditions, or in the geologically complex areas with small target zones, either because of the limitations in data acquisition or restrictions in data processing methods. Alternatively, a Vertical Cable Seismic (VCS) experiment provides a high resolution image for the investigation of the natural resources below the seafloor ${ }^{1)}$. In the VCS configuration, vertical arrays of hydrophones are deployed near the seafloor and are kept in the vertical situation with a buoy moored to the seafloor. A seismic source is towed by a vessel and the data is acquired at every shot point, and then the processing leading down to pre-stack depth migration (PSDM) of the data yields an image of the subsurface structure. However, a good PSDM section would be obtained only if an accurate velocity model becomes available for the migration. Since full waveform inversion (FWI) of the seismic data is a reliable tool to reconstruct the subsurface properties by using pre-stack recorded data, it is worthwhile applying FWI to estimate velocities prior to PSDM.

In this paper we used full waveform inversion of the acoustic waveforms recorded by a VCS simulated experiment to develop $\mathrm{P}$ wave velocity model. Synthetic examples showed FWI could develop reliable velocity models using VCS, even with a few number of hydrophones.

\section{VCS CONFIGURATION}

VCS technique is a novel seismic data acquisition method which is very efficient for high resolution subsurface modeling. Since receivers are very close to the seafloor the signal to noise ratio is very good and reflections from target are recorded with minimum noise contamination. Figure 1 shows a schematic setup of vertical cable. In order to maintain the cable in vertical situation, a buoy on top and an anchor in the bottom fixes the cable. A number of hydrophones at equal intervals are placed on the cable and record the energy propagated through seismic source. 


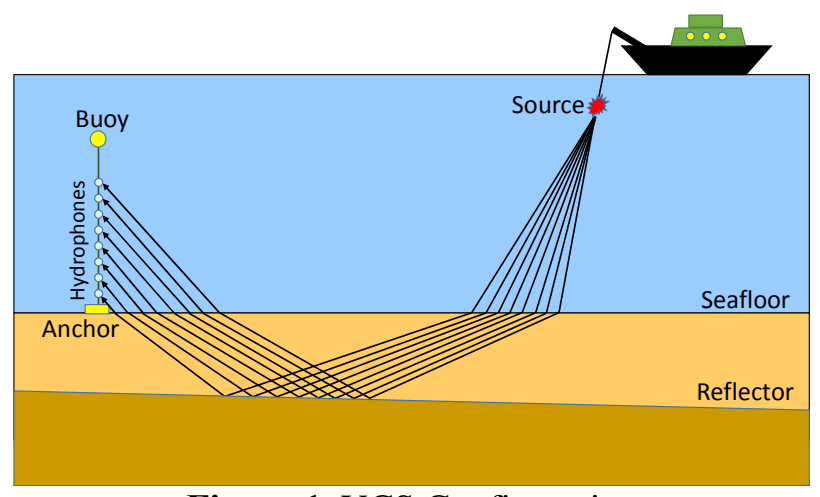

Figure 1. VCS Configuration

VCS has been used for various exploration purposes like subsalt imaging ${ }^{2}$ and sub-seafloor explorations ${ }^{3)}$. Asakawa et al. ${ }^{3)}$ used VCS data to explore Seafloor Massive Sulfide (SMS) deposits around Japan. Their study confirmed promising results by using VCS experiment. Although VCS data acquisition technology has been already developed, data processing methods are not fully defined yet. Because of the especial geometry, VCS data could not benefit from exact conventional surface seismic data processing flows. Some VSP algorithms could work for processing and imaging of VCS data. However, since first arrivals in the VCS data are traveling in water column only, travel time tomography is not able to develop a velocity model similar to what could be obtained in VSP. Because of this problem, we were motivated to evaluate the applicability of FWI on VCS data to produce a reliable velocity model.

\section{FULL WAVEFORM INVERSION}

Full waveform inversion is a local optimization problem which iteratively updates an initial model by minimizing the misfit function, usually defined as the least squares norm of the difference between recorded and calculated data ${ }^{4,5)}$

$$
E(p)=\frac{1}{2} \sum_{j=1}^{N_{s}} \Delta \mathbf{d}_{j}^{t} \Delta \mathbf{d}_{j}^{*}
$$

where, $\boldsymbol{\Delta d}$ is the residual waveform and superscripts $t$ and $*$ denote transpose and complex conjugate, respectively. Summation is over all sources and $p$ is the model parameter. The gradient of the misfit function at $k^{\text {th }}$ iteration for a single frequency is calculated by using adjoint state method as

$$
\mathrm{g}_{k, p}=\sum_{j=1}^{N_{s}} \mathbf{u}_{j}^{t} \frac{\partial \mathbf{A}^{t}}{\partial p} \mathbf{A}^{-1} \boldsymbol{\Delta d}_{j}^{*}
$$

where $\mathbf{u}$ is the modeled waveform and $\mathbf{A}$ is the impedance matrix, i.e. the forward modeling operator. The gradient is scaled with pseudo Hessian matrix and the model parameter is updated as

$$
p_{k+1}=p_{k}-\alpha \mathbf{H}^{-1} \mathrm{~g}_{k}
$$

Step length $\alpha$ is calculated by a line search along the gradient direction using a parabola fitting method. In order to mitigate the nonlinearity of the FWI problem, inversion follows a multiscale scheme from lower to higher frequencies such that the results from lower frequencies are used as initial models for higher frequencies.

\section{NUMERICAL SIMULATION}

In order to evaluate the possibility of using FWI method for subsurface imaging using VCS data we have designed a synthetic example. Based on the real VCS data acquisitions conducted offshore Japan, our desired target is a high velocity anomaly representing Seafloor Massive Sulfide (SMS) deposits in a shallow depth bellow seafloor. Figure 2 illustrates a 3D view of the seafloor topography and $3 \mathrm{D}$ cube of $\mathrm{P}$ wave velocity in the simulation area. We used two perpendicular sail lines, i.e. shot line 1 and shot line 2, for 2D acoustic waveform modeling. The observed data is obtained by frequency domain finite difference solution of wave equation followed by an inverse Fourier transform for time domain data generation. Perfectly Matched Layers (PML) boundary conditions are applied on the edges of the modeling area, including sea surface. Each of 2D shot lines use five vertical cables, with 10 hydrophones at 10 meter intervals in each cable, for recording. Cables are deployed with $200 \mathrm{~m}$ spacing and all of them have the same depth from sea surface such that the top channel is located $390 \mathrm{~m}$ below sea surface. Total numbers of 325 shots are simulated for each line. Time domain traces are displayed in Figure 3, top panel shows shot gather number 163 of line 2 recorded by central vertical cable and bottom panel shows the common receiver gather of top hydrophone at central vertical cable. Clear reflections of layer boundaries are recorded, as Figure $\mathbf{3}$ shows, and we will use these events for FWI. 

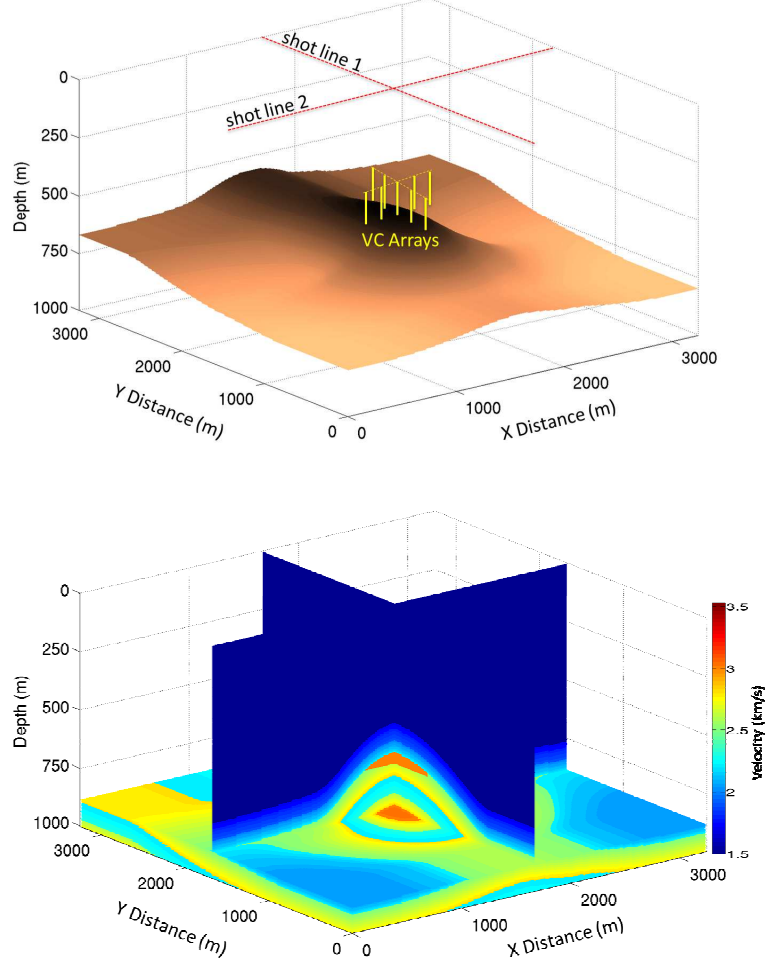

Figure 2. Seafloor topography (top) and $\mathrm{P}$ wave velocity sliced below 2D acquisition lines (bottom).
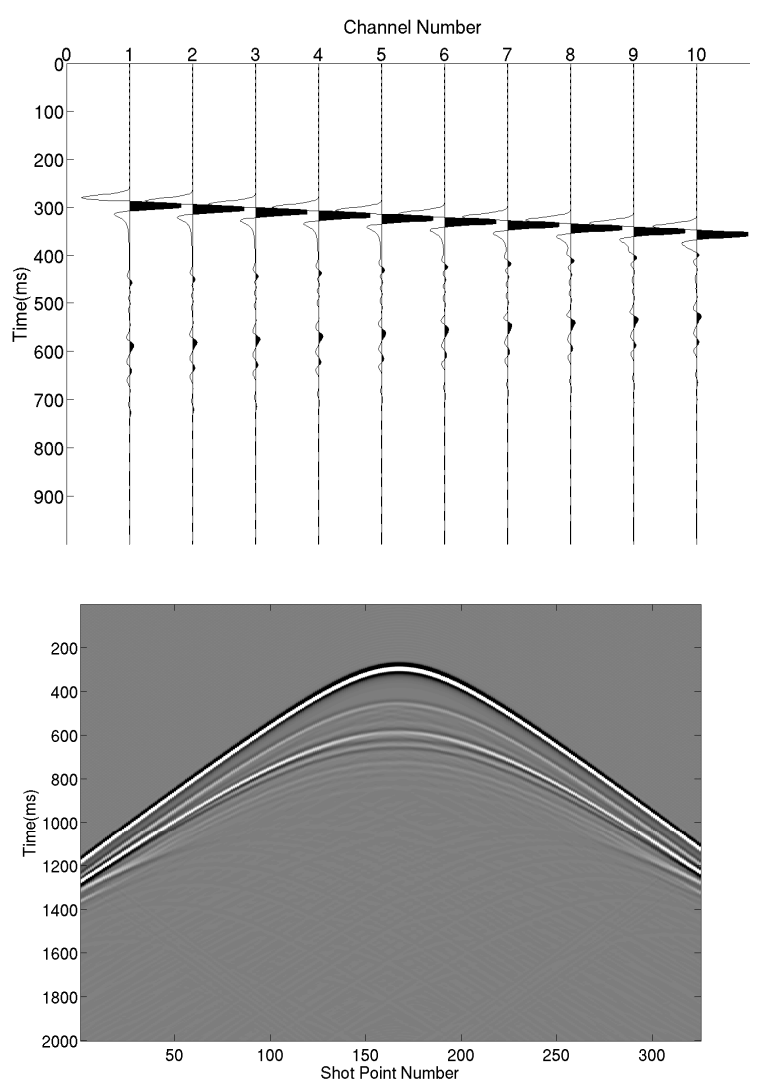

Figure 3. Shot gather number 163 (top) and common receiver gather of top channel (bottom) using central vertical cable from 2D shot line 2 .
Full waveform inversion has been applied on both perpendicular 2D profiles in the frequency domain. The observed data is transformed back to the frequency domain to be used for FWI using a finite number of frequencies. Source wavelet is a Ricker wavelet with dominant frequency of $25 \mathrm{~Hz}$. Total number of 24 frequencies are categorized in eight frequency groups from $2 \mathrm{~Hz}$ to $30 \mathrm{~Hz}$ for FWI via a multi-scale approach. Initial model is a linearly increasing 1D model and 15 iterations of FWI are used for each frequency group. Our full waveform inversion code is developed in MATLAB and benefits from loop vectorization, array processing, and parallel computing for achieving a high performance $^{6}$. Figure $\mathbf{4}$ and Figure 5 show the FWI results of line 1 and line 2, respectively. As expected, because of the especial geometry of VCS experiment, the coverage zone for subsurface imaging is limited to the central part of the simulation area. However, since the desired target is in a shallow depth below seafloor, FWI could detect the target with good accuracy. It should be noted that the water velocity is kept constant from sea surface to depth of $500 \mathrm{~m}$, same as in the initial and true model. As Figure 4 and Figure 5 show, seafloor boundary in the covered area has been properly updated by FWI. Initial residuals, final residuals and normalized misfits for line 2 are shown in Figure 6. Display amplitude scale is same for all grayscale waveform panels in this paper. Direct arrival is automatically excluded from FWI process by using constant water velocity down to depth of $500 \mathrm{~m}$.
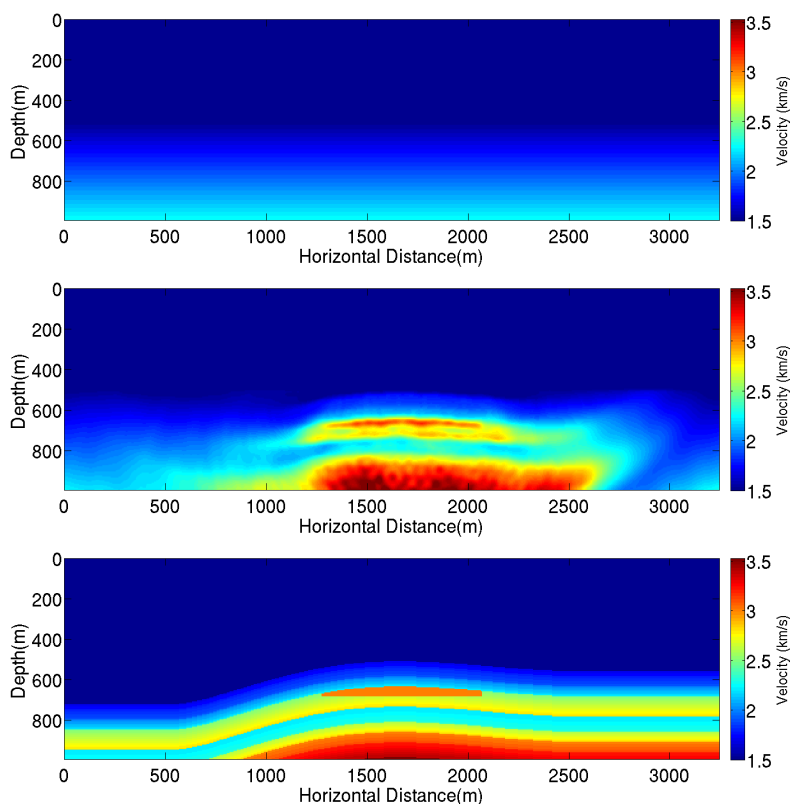

Figure 4. Top to bottom: initial model, FWI result, and true model below 2D shot line 1 . 

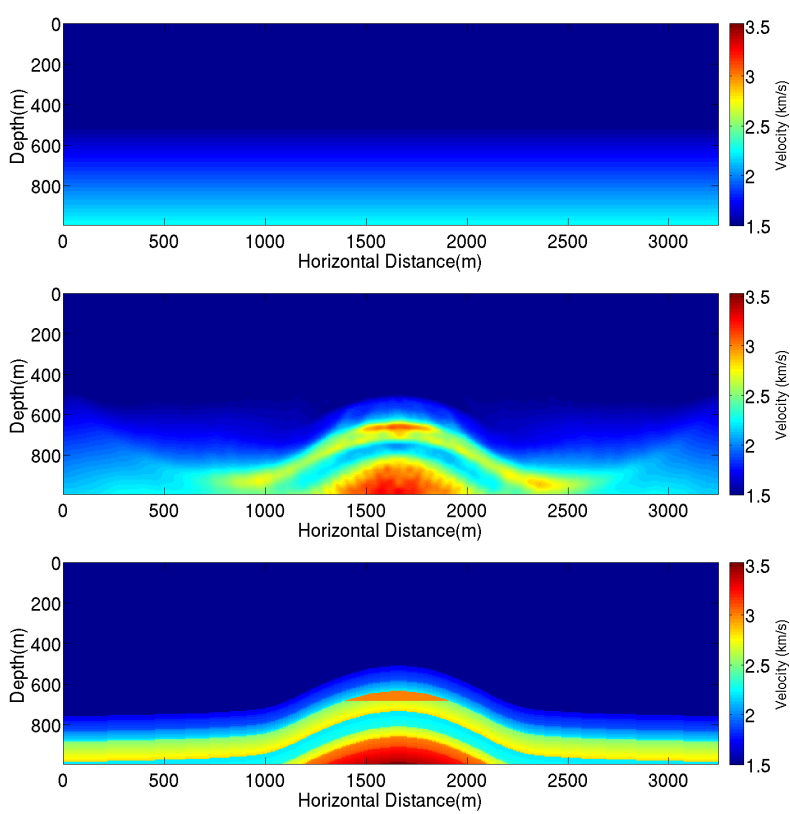

Figure 5. Top to bottom: initial model, FWI result, and true model below 2D shot line 2 .

\section{CONCLUSIONS}

Vertical Cable Seismic (VCS) has been used as an up-to-date technology to acquire high resolution seismic data. As a necessary step for subsurface imaging using this method, a reliable velocity model is required, which could not be obtained using the conventional processing workflows because of the especial geometry of the VCS. Since full waveform inversion is a powerful tool for developing velocity model using observed data, we designed a simulation experiment to detect high velocity anomalies in a shallow depth below the seafloor. These anomalies are considered to be SMS deposits which could be located around Japan. Although the spatial coverage over the area of imaging was strongly affected by the VCS geometry, the results showed that the locations of anomalies could be highlighted. According to the results, we conclude that FWI could be a practical tool used for modeling of sub-seafloor velocities in VCS data. Our future work would be to apply the method to real data to confirm the practicality.

\section{REFERENCES}

1) Asakawa E., Murakami F., Sekino Y., Okamoto T., Ishikawa K., Tsukahara H. and Shimura T., 2012, Development of Vertical Cable Seismic System, 74th EAGE Conference \& Exhibition.

2) KRAIL P.M., 1994, Vertical cable as a subsalt imaging tool, The Leading Edge, 13, 885-887.

3) Asakawa E., Murakami F., Tsukahara H., and Mizohata S., 2014, Vertical Cable Seismic (VCS) Survey for Seafloor Massive Sulphide (SMS) Exploration, 76th EAGE Conference.
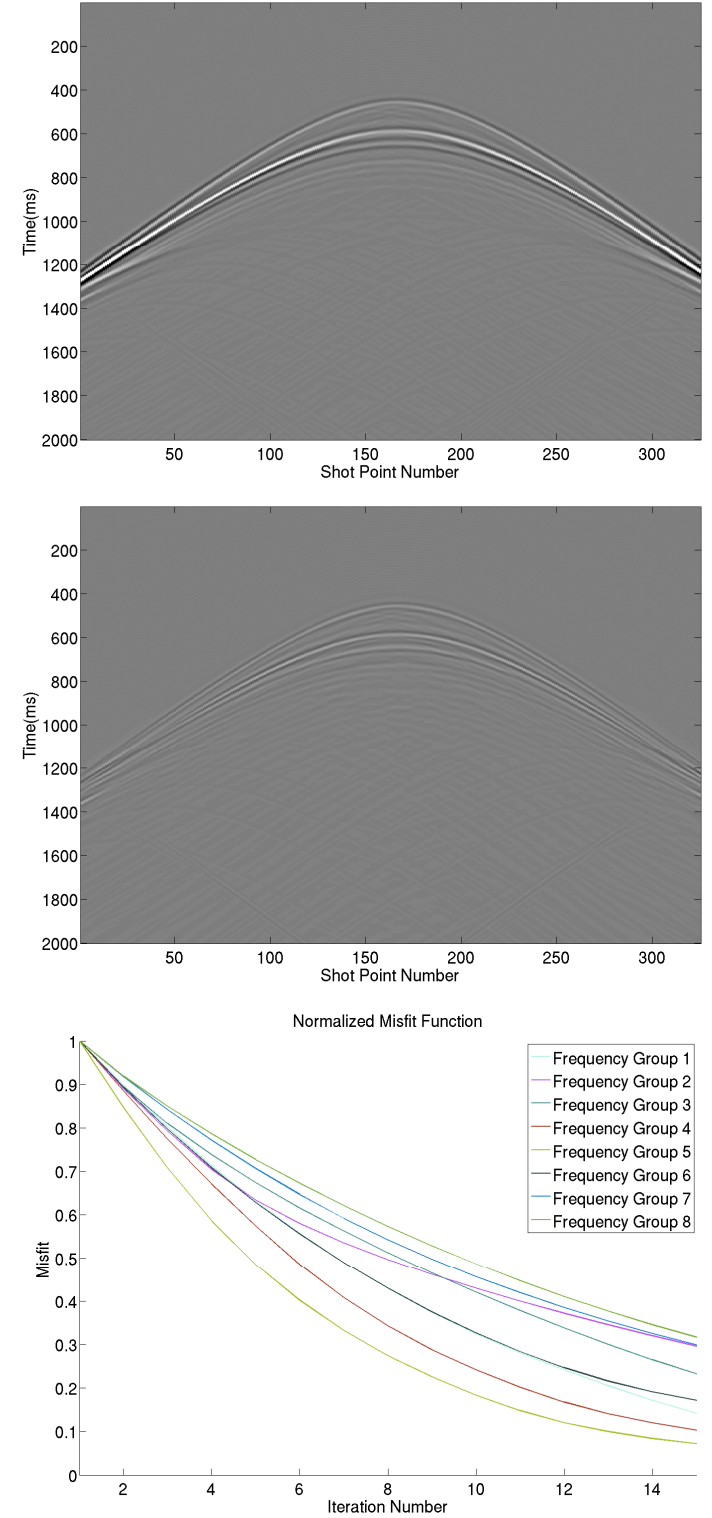

Figure 6. Top to bottom: initial residuals, final residuals, and normalized misfit for FWI results at 2D line 2.

4) Pratt R. G., Shin C., and Hicks G. J., 1998, Gauss-Newton and full Newton methods in frequency-space seismic waveform inversion, Geophysical Journal International, 133, 341-362.

5) Virieux J., and Operto S., 2009, An overview of full-waveform inversion in exploration geophysics, Geophysics, 74, WCC1-WCC26.

6) Jamali Hondori E., Mikada H., Asakawa E., and Mizohata S., 2015, Horizon Guided Initial Model for Full Waveform Inversion of Seismic Data without Low Frequencies, 77th EAGE Conference \& Exhibition. 